\title{
Patient education and the consultation: the importance of lay beliefs
}

Jenny Donovan

In most rheumatology units it is generally accepted that a team approach is the most effective way to treat patients with arthritis. ${ }^{12}$ Patients thus receive medical care from doctors and nurses and from physiotherapists, occupational therapists, social workers, counsellors, etc. Seeing a range of people, patients can obtain different views about their disease and its treatments and thus, some believe, adequate education. For others, however, it is not enough, and education programmes comprising lectures, ${ }^{3-6}$ or metrology/physiotherapy clinics, ${ }^{7-11}$ or group counselling sessions ${ }^{12} 13$ have been established. Evaluating these programmes has always proved difficult, and they have produced some conflicting results. This is perhaps because patient education is not a simple matter of transferring information from team member to patient. Concentrating on implementing programmes emphasises only one side of the story: delivering information. It will be shown below that patients are active participants in the education process and should be given a more central role. ${ }^{14}$

This paper is based on a project set up to investigate information issues in rheumatology clinics. A qualitative methodology was chosen, well established in sociological and anthropological work but unusual in medical research. Individual patients were studied in detail through in-depth interviews in their homes on several occasions so that the complexity of their lives and responses to their illnesses were retained as integral to the research. Because of the intensity of the work only a small number of patients took part: 54 subjects from three rheumatology units in two British cities. The aim was not to produce statistically significant results, but to make sense of arthritis in people's lives and particularly their needs for and responses to information. An important part of the study concerned the consultations between patients and specialist rheumatologists. Each patient was interviewed at length before they came to the clinic to build up a picture of their lives, expectations, perceptions of arthritis, coping strategies, existing levels of knowledge, etc. They were then observed and tape recorded at the first consultation with the doctor and interviewed again shortly afterwards at home. The patients were repeatedly interviewed and observed over periods ranging from six months to three years.

The study produced a vast amount of verbal data. Analysis proceeded slowly through the detailed examination of transcripts of interviews and consultations until themes emerged, which were constantly subject to challenge by the raw data. ${ }^{15}$ These themes were compared with the data until they became drawn together to produce results and theory.

\section{The patients}

Patients with possible rheumatoid arthritis were selected by consultant rheumatologists from letters of referral by general practitioners. Fifty four patients were selected, and of these, 40 had an inflammatory arthropathy of some kind and 30 rheumatoid arthritis. The others had osteoarthritis, simple aches and pains, viral illness, etc. In the interviews before they arrived at the clinic patients disclosed a wide variety of ideas about their joint problems. Many of these were complex and detailed perceptions and theories about, for example, the onset of symptoms, cause, treatments. They can be termed 'lay beliefs', rooted as they are in common sense, family traditions, and folklore. Lay beliefs, studied in sociology and anthropology, ${ }^{16}{ }^{17}$ have been shown to lie behind much of people's behaviour concerning health and illness.

Many patients had learnt to live with their joint problems for months or years and considered them 'just part of life', an inconvenience that they put up with and lived around. For a small number (seven) the arthritis was an 'illness' that totally disrupted their lives. Most had worked out a theory of cause based on common sense, such as heredity, cold, or damp weather. It was abundantly clear that their symptoms were always put into a context. They did not talk of degrees of pain or stiffness, but of pain which prevented them working or doing hobbies, or described pain and stiffness using metaphors ('like toothache gnawing all the time' or 'like hot coals'). As symptoms are nearly always contextually based this has implications for the validity of pain scales such as the visual analogue. People have great difficulty measuring pain within such artificial constraints and out of context.

Lay beliefs also affect the ways patients cope with disease. The seven who felt overpowered by the illness were unable to draw on these, seeming to succumb to a feeling of bereavement for the life they had had before the onset and dwelling on their symptoms, unable to think of anything else. The others, however, had a range of strategies for coping. Eleven (all women) fought the disease and suffered considerably in their efforts to 'keep going'. They ignored all advice to rest (given later by most members of the team), believing that this would be 'giving in', 'letting the arthritis win'. Most of the others coped by changing the ways they did things: 
doing a different job, slowing down, changing priorities, asking others to help, etc. All were inundated by well meaning advice from all quarters: friends, relatives, magazines, and half had tried alternative treatments, such as acupuncture, fish oils, special diets, and so on. These substituted for or supplemented general practitioner prescriptions. Each patient balanced the costs and benefits of each treatment as they understood it, and many wanted to avoid the side effects (real or imagined) of drugs. They believed alternatives to be free from side effects.

Most patients expected the clinic to diagnose their problems and to suggest treatments. Most of them were going to the clinic with well worked out and internally consistent sets of lay beliefs. Some of these accorded with medical theories, but where they did not, there would be opportunity for conflict. Three patients wanted the doctor to take total control over their treatment. Most wanted to hear the doctor's diagnosis and advice before deciding whether they would make use of it or not. Patients do not just accept what they are told: it has to make sense and be justifiable within their way of looking at the world.

\section{The consultations}

The clinics observed were busy NHS clinics. Consultations varied in style between doctors and depending on such things as the mood of the doctor, pressure of work, rapport between doctor and patient, number of previous meetings, mood of the patient, and so on. There were, however, some clear patterns. Often, doctors gave similar explanations for particular conditions or tests. Almost all first consultations followed the same basic pattern of three distinct phases: history taking, physical examination, explanation, though they varied in length from 10 to 35 minutes. At patients' second visits doctors provided much more information as blood tests, radiographs, and other results were delivered and explained. Later routine follow up visits were progressively less formal; almost a conversation between friends about current state of health or particular problems. Others have found similar sorts of patterns. ${ }^{18} 19$

\section{Patient education: does it happen in consultations?}

The intensive and in-depth nature of the study allows a close analysis and understanding of how information is delivered by doctors and received or used by patients. It is interesting to look at patients' first meetings with rheumatologists because these represent the first access patients have to specialist information about diagnosis and treatment.

The history taking phase of the first consultation consisted primarily of the doctor asking a set of closed questions - that is, ones to which the answers were confined to yes or no or a few words-for example, 'Any asthma in the family?'. Occasionally, patients took the initiative, but on the whole, doctors maintained their control by using closed questions, interrupting, or abruptly changing topic. In the second phase little was said as the doctor did the routine physical examination. The third phase was characterised by the doctor speaking for some time without pause. The amount of information conveyed depended upon many factors: confidence in the diagnosis, mood of the doctor, pressure of the clinic, and the doctor's impression of how much the patient wanted to know, for example. The tone of the doctor's delivery was markedly different depending on the diagnosis. Those with suspected inflammatory conditions (rheumatoid arthritis, ankylosing spondylitis, etc) or interesting unusual problems were treated more sympathetically and at greater length than others because they were likely to become long term patients and were seen to be deserving of the doctor's attention.

On the whole, consultations seemed to go smoothly and to follow clear routines. Doctors were always active: taking the history, doing the physical examination, giving the explanation. Patients were nearly always passive or reactive: answering questions, being examined, listening to the explanation. In most cases substantial amounts of information were given by doctors about the diagnosis (when it was clear), possible treatments, cause of symptoms, possible future outcome. It was one of the main purposes of this study to discover how much of this information patients absorbed and were willing to use.

Most patients (29) said that they were satisified overall with their visits, but most had some complaints, such as long waiting times or feeling rushed. Ten were critical of almost all aspects; another 13 were happy about almost everything. Patients found it easiest to comment about the doctor in personal terms: the most satisfied saying $s /$ he was kind or caring, and the most dissatisfied that $\mathrm{s} / \mathrm{he}$ was clinical or matter of fact. Patients particularly appreciated doctors who seemed to take their time and were most critical of those who appeared rushed or interrupted them. Indeed, the most satisfied patients did have, on average, longer consultations than the very dissatisfied.

Few of the patients had prior experience of hospital consultations, and in the interviews before they attended the clinic most expressed anxiety about their disease, about what might happen at the clinic, and showed that they were in awe of the specialist whom they had waited weeks or months to see. After their appointments they said that they felt unsure of the routines of the clinic and wondered if they had said the right things-more than half felt they had omitted important details and one third believed the doctor had not understood the severity of their problems.

An interesting example of the differences in perceptions of clinic routines is given by the physical examination. To medical staff the full physical examination was an expected part of the routine of investigating a rheumatic complaint. To more than one third of the patients, however, it was the most surprising aspect of their visit. Some found it embarrassing, others thought it unnecessary when their symptoms were confined to their hands. Because it is such an integral part of clinic routines the physical 
examination was usually just announced and its purposes were rarely, if ever, explained to patients.

Patients did not always understand the information given by doctors in the way that it was intended. Patients wanted doctors to understand the severity of their problems as they perceived them within the context of their everyday lives. Doctors, however, tended to see symptoms more in terms of biomedical criteria. Doctors tried to reassure patients and give them a positive outlook and so routinely said that the patient's disease was mild and in the early stages. Compared with other patients, this was often the case, but for individual patients it did not make sense. In their terms they were in considerable pain, might have had deformities, and feared for the future. The idea that this was the 'mild' form of the disease or at its early stages merely heightened their anxieties and fears for the future-the opposite of what was intended.

Many studies have concentrated on patient recall as a measure of the success of consultations. ${ }^{20}$ In this study, however, it can be seen that the ability to recall information does not necessarily mean that patients will either accept or make use of what they can recall. One half of these patients could recall in detail almost everything that happened in the consultation, and one third could recall most things but forgot some details. But nearly three quarters of those who could recall almost everything either disagreed with or rejected what the doctor had said. For these patients the doctors' diagnoses and advice failed to concur with their own thoughts and opinions (lay beliefs).

As an example, one patient had been told by his general practitioner that he had rheumatoid arthritis. After reading books and talking to relatives he came to the conclusion that he had gout. When he went to the clinic the registrar told him he had ankylosing spondylitis and explained in great detail about the disease and its treatments. The patient spent some time on the ward receiving instruction about exercise and was given two booklets. He remained, however, convinced that he had gout. He justified this by pointing out several symptoms of ankylosing spondylitis that he did not have, declared that doctors could not agree anyway, and added that he would not do 'a lot of boring exercises for the rest of my life', especially when he was not convinced that they would help. Being young and shy, the patient did not clearly express his beliefs to clinic staff, and because they went undetected, he was thought to be non-compliant, unintelligent, and lazy.

Although consultations largely proceed smoothly and without overt conflict, they do not easily facilitate the transfer of information from doctor to patient and vice versa. They are formal events with set routines. Doctors feel 'at home' in familiar surroundings, are usually relaxed and, for them, the consultation is just part of working life. For patients, however, consultations are very different and difficult experiences. Patients are usually unaware of routines, anxious about their illness, in awe of the doctor, and then find they have to talk about intimate details and undress for a physical examination. In the interviews with the researcher at home patients were keen to talk about all aspects of their illess, but in consultations they tended to be hesitant and quiet. Despite their intentions to ask questions, few managed any, even though most were given the opportunity at the end of the consultation. One of them explained: 'He put all the notes away and is half way through the door and says, "Any questions?" before I really had the chance to think of any ... You are still trying to sink in what he has told you.'

Patient education: How can it be done?

Educating patients effectively about their disease and its treatments is extremely difficult. Doctors and patients approach consultations from different perspectives, with different views of illness and its severity, and often with different sorts of knowledge about health and illness. Doctors and patients also tend to differ socially: whereas patients span the spectrum, doctors are still mainly white, upper-middle class men who communicate best with similarly educated peers. ${ }^{21} 22$ Consultations tend to be one sided, controlled by doctors, ${ }^{23}$ and the patients in this study found it difficult to tell their stories. As a consequence their lay beliefs, which are so important in determining behaviour, remained largely hidden from the medical profession.

Any advice patients are given (lay or medical) has to pass through the filter of their lay beliefs. Occasionally, medical and lay views coincide and patients are happy to accept the doctor's advice and treatment. At other times they clash and patients can be left confused, dissatisfied, and sometimes critical of the doctor. Patient beliefs may be different from biomedical theories, but they are usually internally consistent and rational in their own terms. They are not static, but change in the light of new experiences and the availability of believable information. They can adopt scientific ideas but only if they are credible (viruses are now as much common sense as medical theory).

Patient behaviour is determined by several factors. People weigh up the costs and benefits of various options open to them before choosing the most suitable. Medical advice thus has to compete with a range of other suggestions, and if not credible, may be dismissed. People's lay beliefs determine what advice is reasonable, but actions also have to be possible within the constraints of everyday life. Some patients may reject advice to rest because it does not make sense-it makes them stiff. Others may reject it because of family commitments, such as demanding children. It is important to elicit lay beliefs to understand reasons for apparent noncompliance.

Many doctors are becoming aware that the consultation is not the most effective place for patient education. As shown above, its formality and impenetrable routines make it difficult for patients. As patients are increasingly demanding of information the response of many caring rheumatology teams has been to set up didactic 
education programmes. Lectures are a familiar format for medical staff, but for most patients are an alien and uncomfortable experience. They maintain the dominance of medical staff, ignore patients' lay beliefs, and although they claim to increase patient knowledge, there is no guarantee that this will, in itself, have any effect on behaviour.

Some rheumatology teams have introduced other ways of educating patients, through clinics led by specialist nurses or physiotherapists. These move away from the fixed routines of consultations and as patients feel less in awe of non-doctors, these may prove to be more successful. Group sessions have also been tried, but they have produced conflicting results. This might be because some patients will find such sessions enormously helpful (Arthritis Care has thrived on active groups), but others may find them as difficult as lectures or consultations.

The clearest finding from this study to date has been that most patients want to be more active participants in their treatment. Most want more information about such things as the cause of arthritis, diagnosis, reasons for and results of tests, prognosis. They are particularly concerned to know about a wide range of treatments-drugs, physiotherapy, and alternatives. Most want to know more about the drugs they take: why they have been given a particular drug, what side effects they might have, for how long they should take them. If they do not get the information they want patients decide whether or not to comply based on their lay beliefs and what they have managed to glean (accurate or not).

Up to now the emphasis in many medical specialties, including rheumatology, has been on educating patients. This implies that patients have little knowledge of their own and need instruction. ${ }^{14}$ This intensive study of patients' views has shown that there needs to be a change of emphasis. On the whole, patients are not ignorant and lacking in ideas and beliefs. Far from it: they have detailed and well developed lay beliefs, which may or may not concur with medical theories, and which greatly influence their behaviour. Many want more medical information and to become more equal participants in their treatment. There will always be some who want the doctor to take total charge, but these are a very small proportion.

What is needed, then, is information provision. Information about all aspects of arthritis and its treatments should be made available to patients so that they can choose what to make use of in order to make informed decisions about their health. It needs to be given in jargon-free language and in informal ways that are separate from, but complement, medical consultations. Leaflets can be useful, but a sympathetic person (not a doctor), who could spend some time with patients discovering their lay beliefs and explaining about arthritis and treatments, would be better. Provision of information for patients could then become an important and integral part of treatment by the rheumatology team, and patients would be better able to make informed choices about their treatment.

The project on which this paper is based was funded by the Arthritis and Rheumatism Research Council.

I Moll J M H. Medical communication. In: Moll J M H Sturrock R D, eds. Recent advances in rheumatology 4. Bath: Churchill Livingstone, 1986: 73-102.

2 Winfield J. Current approaches to the rehabilitation of the rheumatic patient. In: Moll J M H, Sturrock R D, eds. Recent advances in rheumatology 4. Bath: Churchill Living stone, 1986: 211-34.

3 Vignos P J, Parker W T, Thompson H M. Evaluation of clinic education programme for patients with rheumatoid arthritis. F Rheumatol 1976; 3: 155-65.

4 Stross J, Milkkleson W. Educating patients with osteoarthritis. F Rheumatol 1977; 4: 313-6.

5 Kaye $R$, Hammond A. Understanding rheumatoid arthritis ҰAMA 1978; 239: 2466-7.

6 Spiegel T M, Knutzen K L, Spiegel J S. Evaluation of an inpatient rheumatoid arthritis education program. Clin Rheumatol 1987; 6: 412-6.

7 Katz S, Vignos P J, Moskowitz R W, et at. Comprehensive out-patient care in rheumatoid arthritis. $\mathcal{F} A M A$ 1968; 206: 1249-54.

8 Vignos $\mathrm{P}$ J, Thompson $\mathrm{H} M$, Katz S, et al. Comprehensive care and psychosocial factors in rehabilitation in chronic rheumatoid arthritis. $\mathcal{F}$ Chronic Dis 1972; 25: 457-67.

9 Gallez P. Rheumatology health education. Nursing Mirro 1985; 160: 37-9.

10 Hill J. Nursing clinics for arthritics. Nursing Times 1985; Sept 18, 33-4.

11 Wright $\mathrm{V}$, Hopkins $\mathrm{R}$, Jackson $M$. Instructing patients in physiotherapy. Rheumatology and Rehabilitation 1980; 19: 91-4.

12 Shearn M, Fireman B. Stress management and mutual support groups in rheumatoid arthritis. Am Med $\mathcal{f} 1985$; 78: 771-5.

13 Kaplan S, Kozin F. A controlled study of group counselling in rheumatoid arthritis. $\mathcal{F}$ Rheumatol 1981; 8: 91-9.

14 Donovan J, Blake D, Fleming W. The patient is not a blank sheet: lay beliefs and their relevance to patient education. Br $\mathcal{F}$ Rheumatol 1989; 28: 58-61.

15 Glaser B, Strauss A. Grounded theory. Chicago: Aldine, 1967.

16 Cornwell J. Hard earned lives. London: Tavistock, 1984.

17 Donovan J. "We don't buy sickness, it just comes": health and illness in the lives of black people in London. Aldershot: Gower, 1986.

18 Byrne P, Long B. Doctors talking to patients. Exeter: HMSO, 1976.

19 Fisher S. Doctor patient communication. Sociology of Health and Illness. 1984; 6: 1-29.

20 Ley P, Spelman M. Communicating with the patient. London: Staples Press, 1967 .

21 Helman C. Communication in primary care. Soc Sci Med 1985; 20: 923-31

22 Bochner S. Doctors, patients and their cultures. In: Pendleton D, Hasler J, eds. Doctor patient communication. London: Academic Press, 1983: 127-38.

23 Mishler E. The discourse of medicine. New Jersey: Abler, 1984. 